\title{
The Straightness detecting of deep pipe
}

\author{
Wang Weiguo ${ }^{1,2, a}$, Li Juguang ${ }^{3, b}$ and Du Jie ${ }^{3, b}$ \\ ${ }^{1}$ 1Beijing Institute of Technology No.5 Yard, Zhong Guan Cun South Street \\ Haidian District, Beijing, China 100081 \\ 2 Shijiazhuang Mechanical Engineering College, Shi Jiazhuang, China 050003 \\ ${ }^{3}$ ShiJiazhuang Mechanical Colleage, Shijiazhuang, China 050003 \\ a wangweiguohh@yahoo.com.cn, ${ }^{b}$ lijuguang@126.com, 'machunt@163.com
}

Keywords: straightness measurement CMOS lens optoelectronic system .

\begin{abstract}
Based on the laser straight line dissemination rule, an optoelectronic measuring method is presented to measure straightness of the deep pipe with a diameter of $30 \mathrm{~mm}$ by using the quadrant detection, precision mechanical structure, image processing and SQ606 processor. The overall structure of system is discussed, and the measuring principle of the system is analyzed. Then, the corresponding formula to calculate the straightness of the deep pipe is alse discussed. The system is used to measure a deep pipe with the length of $1.15 \mathrm{~m}$ and $30 \mathrm{~mm}$ inner diameter. Finally, the straightness curve of whole deep pipe is given by data fitting. The results show that the system resolving power can reach $0.01 \mathrm{~mm}$, which indicates that the measuring method is satisfied to the detecting system.
\end{abstract}

\section{Introduction}

Straightness of the deep pipe immediately concerns reliability, service life and usable performance of associated mechanical structure, so the high precision measuring of straightness is vitally necessary. Now, laser Doppler method, EST measurement and quadrant detection method are presented to measure straightness of the deep pipe at home and abroad. When using quadrant detection method, laser is the straight line of dissemination, straightness measurement is based on laser position in relation to quadrant detection instrument. By using the quadrant detection method, precision machine, image processing and SQ606 processor, a non-destructive method is presented to measure straightness of deep pipe.

\section{The Overall Structure of Measuring Instrument System}

The instrument contains centering adjusting mechanical structure, laser, optoelectronic system, CMOS lens imaging system, computer and data processing system.

By using centering adjusting mechanical structure, lasers and optoelectronic system is installed the ends of the deep pipe, laser is took to optoelectronic system. The straightness of whole deep pipe is obtained by CMOS lens and data processing system, at the same time, data file, storage, display, printout controlled by the SQ606 processor. The overall structure is shown in Figure 1. 


\subsection{Laser system}

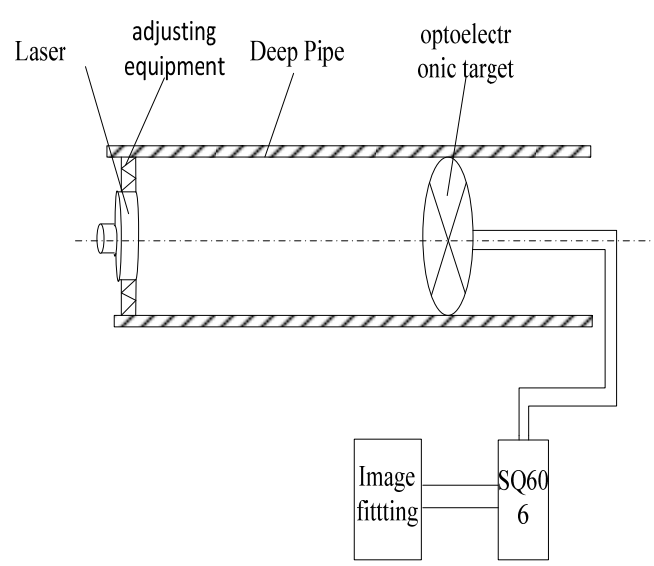

Figure 1 The overall structure of detectiong system

The system uses $5 \mathrm{~mW}$ He-Ne white light laser, some characteristic features which contains is as follows:

1.Working substance is He-Ne mixed gas, which is solid color and a quality of uniformity. The laser is great coherent source, the power output and wavelength is stability controlled.

2.Laser is nearly straight line, which has great directional. The laser shot out in an angle of only a few radian.

3. The laser contains many advantages: simple structure, small volume, light in weight, long life.

1.2 Centering adjusting mechanical structure

To ensure the rigid overlapping the central point of laser and deep pipe, the central point of laser and optoelectronic system must be in the axis of deep pipe, so centering adjusting mechanical structure is used to adjust the placement. The figure 3 is the centering adjusting mechanical structure.

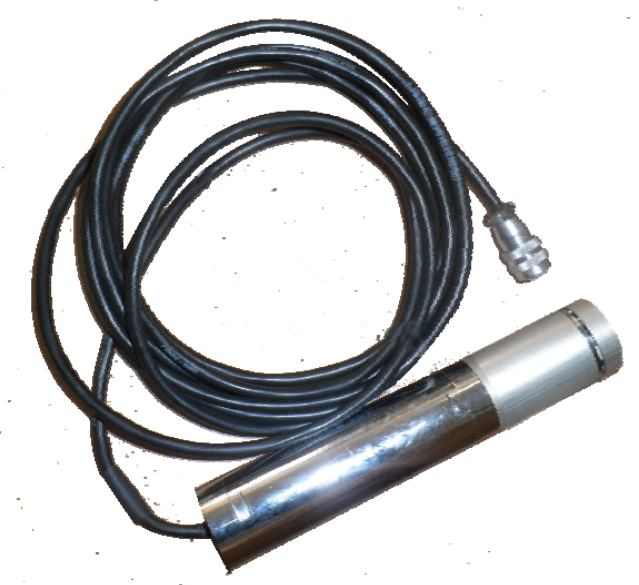

Figure 3 The photograph of the mechanical structure

When designing centering adjusting mechanical structure, there are two main factors:

1. How to glazing centering adjusting mechanical structure in the deep pipe, at the same time, to screwing it.

2. To surmount gravity of centering adjusting mechanical structure, ensuring it in the center of deep pipe.

Therefore, centering adjusting mechanical structure is designed to be elastic element. The circular ring substance lodges the inner layer of deep pipe, simultaneously, three springs at interval of $120^{\circ}$ is glazed in the centering adjusting mechanical structure, to ensure centering adjusting mechanical structure in the center of the deep pipe.

1.3 Optoelectronic target

The main parts of optoelectronic system is OV9650 CMOS lens camera that is made by OmniVision, The OV9650 CAMERACHIPTM is a low voltage CMOSimage sensors that provides the full functionality of asingle-chip SXGA (1280x1024) camera and image processor in a small 
footprint package. The OV9650 provides full-frame, sub-sampled or windowed 8-bit/10-bit images in a wide range of formats, controlled through the Serial Camera Control Bus (SCCB) interface.

The OV9650 product has an image array capable of operating at up to 15 frames per second (fps) in SXGA resolution with complete user control over image quality, formatting and output data transfer. All required image processing functions, including exposure control, gamma, white balance, color saturation, hue control, white pixel canceling, noise canceling, and more, are also programmable through the SCCB interface. In addition, OmniVision CAMERACHIPS use proprietary sensor technology to improve image quality by reducing or eliminating common lighting/electrical sources of image contamination, such as fixed pattern noise, smearing, etc., to produce a clean, fully stable color image.

\section{The principle and mathematical model of laser straightness tester}

Laser is placed on the one side of the deep pipe, as the standard axis, the laser is shoot to the other side on which there is the area of CMOS lens, then, to ensure the information of the row and line about the laser spot center. By calculation, the coordinate of geometric center is got. Then, citing the center of optoelectronic system for comparison, the deviation of coordinate value is got by signal process. Using the same method, the reality center of many sections can be got. Thus the straightness of the deep pipe is gained, then, the curves of straightness can be got by data fitting.

For ease of the theoretical analysis, the mathematical model is shown in Figure 3. the laser certre photograph is shown as fiure 4

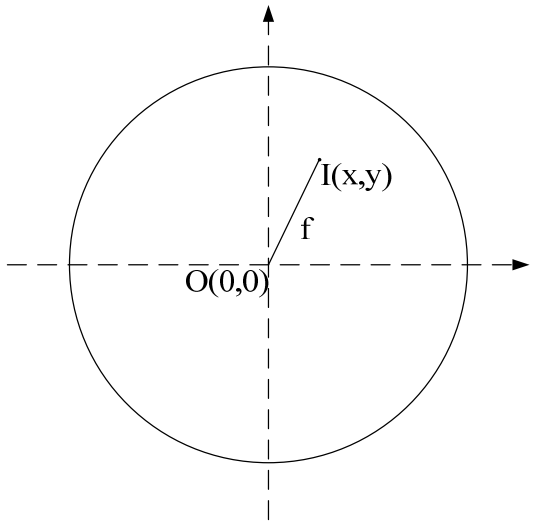

Figure 3 The mathematical model

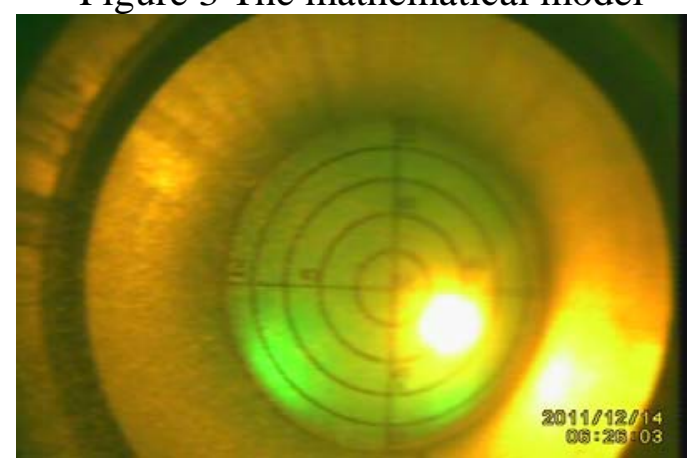

Figure 3 The photograhp of the laser centre

Coordinate system XYZ is established on the useful active area, the origin of coordinate is centroid of the matrix CMOS lens, as the standard of the origin. By data processing and image processing, the laser spot center $(\mathrm{x}, \mathrm{y})$ in the CMOS lens floater is figured out. Then, comparing with the origin, the $\mathrm{f}=\sqrt{\mathrm{x}^{2}+y^{2}}$ eccentricity between actual center and origin. the computer assessing algorithm is shown in Figure 5: 


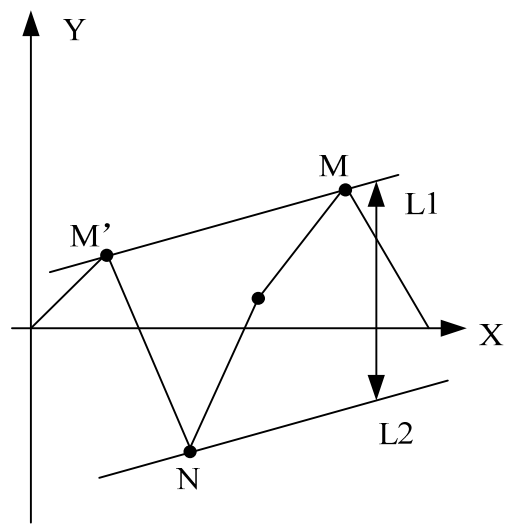

Figure 5The computing assessing algorithm

Step1. Judge whether accordant head and the tail of error curve, yes, turn to step2; no, making the curve become contour.

Step2. Find out the lowest point and the highest point in the curve.

Step3. Make two straight L1 and 12 line parallel X axis through M, N.

Step4. L1 reverse by $\mathrm{M}$ point andL2 reverse by $\mathrm{N}$ point, in the same direction, until one of them touches the error curve, the point $\mathrm{M}^{\prime}$ is the second highest point.

Step5. Judge whether the three points $\mathrm{M}, \mathrm{N}, \mathrm{M}^{\prime}$ are alternated, yes, turn to step6; no, $\mathrm{M}^{\prime}$ takes the place of $\mathrm{M}$, then, turn to step3.

Step6. Calculate the distance between L1 and L2, this distance is the straightness error.

\section{The results analysis and result}

Using a pipe 1metres in length and $30 \mathrm{~mm}$ in diameters, every group takes a sample that contains 10 points, step is $100 \mathrm{~mm}$.By software, SQ606 processor based on least-squares curve fitting is used for data fitting. The fitting curve is shown in Figure 6.

$\mathrm{X}$ axis is the eccentricity between the center of section and the center of theory; $\mathrm{Y}$ axis is the number and distance of sampling. The experiment results, the resolving power of measurement system can reach $0.01 \mathrm{~mm}$.

Straightness testing system for deep pipe solves the difficulty about nondestructive testing of de deep pipe in the abominable environment, ensuring the mechanical structure reliability. The testing system contains fast speed and high accuracy, at the same time, the system can realize control in real time and data processing, which supply the requirement of deep pipe nondestructive testing, so this system is application value and popularization value

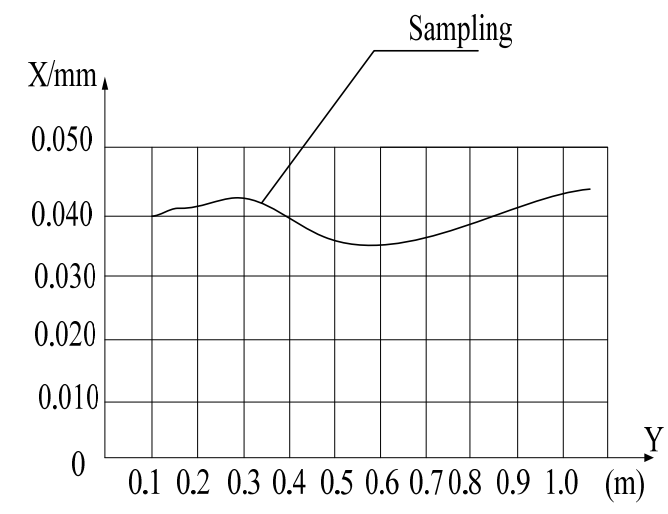

Figure 6The fitting curve

\section{References}

[1] Zhang guoan, An zhiyong. Optoelectronic non contact measuring method for straightness error

[J]. Optical Technique, 2008,25(1): 41-43. 
[2] Wang quandou, Yu jingchi. Application of Phase Shift Interferometry to the Measurement of Small Angle and Linearity [J]. Acta Metrologica Sinica, 2002,23(1):16-9

[3] Zhou weiqing, Ma hong. Development of an Instrument for Straightness of Bore[J], SQ606 PROCESSOR MEASUREMENT \& CONTROL, 2006.14(7):929-958

[4] Cheng kaifu. Development Status and Application of CCD Image Sensors. Semiconductor Optoelectronics. 2003,3(2): 25-28.

[5] SQ606B_CCTV_ASICBrief_V1_0_2009 0114.pdf www.sq.com.tw 